\title{
Risk factors of diabetic foot Charcot arthropathy: a case-control study at a Malaysian tertiary care centre
}

\author{
Aishah Ahmad $\underline{\text { Fauzi }}^{1}$, MBBS, MRehabMed, Tze Yang $\underline{\text { Chung }}{ }^{1}$, MBBS, MRehabMed, Lydia Abdul $\underline{\text { Latif }}^{1}$, MBBS, MRehabMed
}

\begin{abstract}
INTRODUCTION This study aimed to determine the risk factors of diabetic Charcot arthropathy of the foot among diabetic patients with and without foot problems.

METHODS This was a case-control study involving diabetic patients attending the Diabetic Foot Care and Wound Management Clinic at University Malaya Medical Centre, Kuala Lumpur, Malaysia, from June 2010 to June 2011. Data on sociodemographic profiles, foot factors and diabetes characteristics was collected and analysed.

RESULTS A total of 48 diabetic patients with Charcot arthropathy of the foot were identified. Data from these 48 patients was compared with those of 52 diabetic patients without foot problems. Up to $83.3 \%$ of patients with diabetic Charcot arthropathy presented with unilateral Charcot foot, most commonly located at the midfoot (45.8\%). Patients with a history of foot problems, including foot ulcer, amputation, surgery or a combination of problems, had the highest (26-time) likelihood of developing Charcot arthropathy (odds ratio 26.4; 95\% confidence interval 6.4-109.6). Other significant risk factors included age below 60 years, more than ten years' duration of diabetes mellitus and the presence of nephropathy. CONCLUSION A history of prior diabetic foot problems is the greatest risk factor for developing diabetic Charcot arthropathy, compared with other risk factors such as diabetes characteristics and sociodemographic profiles. Preventive management of diabetic foot problems in the primary care setting and multidisciplinary care are of paramount importance, especially among chronic diabetic patients.
\end{abstract}

Keywords: Charcot arthropathy, diabetes mellitus, diabetic foot problems, risk factors

\section{INTRODUCTION}

Diabetic Charcot arthropathy is a debilitating condition that is associated with peripheral neuropathy and characterised by the progressive destruction of weight-bearing bones and joints, which leads to gross instability, recurrent ulcerations and/or amputation. ${ }^{(1)}$ The estimated prevalence of diabetic Charcot arthropathy, as reported in various studies, ranged from $0.08 \%$ in the general diabetic population to about $13 \%$ in specialty diabetic foot clinics. ${ }^{(2)}$ The prevalence rate is believed to be variable, as patients remain undiagnosed due to clinicians' lack of training and inadequate understanding of the natural history of diabetic Charcot arthropathy. ${ }^{(3)}$

Charcot arthropathy is a complication of diabetes mellitus that further increases the morbidity and mortality of patients. ${ }^{(4,5)}$ A long-term follow-up study has shown that longstanding Charcot foot will decrease a patient's physical functions and quality of life. ${ }^{(6)}$ Peripheral neuropathy is believed to be the prerequisite for the development of Charcot arthropathy, although not all diabetic patients with peripheral neuropathy will develop Charcot joint. ${ }^{(7)}$ Theories on the pathogenesis of Charcot arthropathy have evolved from the interaction of the neurotraumatic and neurovascular theories to the current, much more well-recognised inflammatory theory. ${ }^{(8-10)}$ These theories help to explain why Charcot arthropathy often occurs unilaterally at first onset, whereas peripheral neuropathy has symmetrical involvement. However, the pathogenesis and aetiology of diabetic Charcot arthropathy remain unclear; thus, independent predictors of this disease should be identified among the diabetic population in order to provide adequate and specific measures to prevent this debilitating condition.

According to previous reports, the risk factors for diabetic Charcot arthropathy are multifactorial. Obesity with peripheral neuropathy, comorbid renal failure, being of an older age group (> 65 years) and race (white) have been reported as significant predictors of diabetic Charcot arthropathy in a large cohort study. ${ }^{(11)}$ Other studies reported that diabetic patients who are ambulant, and have nephropathy, microvascular complications (peripheral neuropathy and retinopathy), and a history of previous foot ulcer are significantly prone to developing diabetic foot Charcot arthropathy. ${ }^{(12,13)} \mathrm{A}$ history of preceding trauma, which includes diabetic foot problems (foot ulcers, foot deformities, prior foot complex surgery or amputation), may be a factor that triggers the events leading to the development of Charcot arthropathy. ${ }^{(2,14)}$

In view of these multifactorial predictors, this study aimed to obtain data on the risk factors of diabetic Charcot arthropathy in Malaysia's multiracial society. Information on the predictors of diabetic Charcot arthropathy may help medical practitioners in primary care as well as other physicians to better address the needs of diabetic patients, and to provide early detection for appropriate referrals and expert management.

\section{METHODS}

This was a hospital-based case-control study conducted over a one-year period (from June 2010 to June 2011) at University 
Malaya Medical Centre (UMMC), Kuala Lumpur, Malaysia. The case and control groups were taken from the same population at the Diabetic Foot Care and Wound Management Clinic, a specialised clinic for foot-care education and monitoring that serves not only diabetic patients with foot problems (i.e. Charcot arthropathy, foot deformities and ulcers), but also those without foot deformities who may or may not have peripheral sensory neuropathy. Patients are referred from various departments, including primary care medicine, endocrine and orthopaedic clinics.

The case group included 48 diabetic patients with Charcot arthropathy of the foot, who were diagnosed by the attending physician. The control group, who were recruited from the same clinic, comprised 52 diabetic patients without Charcot arthropathy, ulcer or other foot deformities. All 100 patients involved in the study had Type 2 diabetes mellitus. The universal sampling method was used to gather all diabetic patients with Charcot foot deformities (case group) and those who had no foot deformities (control group), until the minimum number was achieved, based on the sample size estimation, within the stipulated timeframe. The independent variables used to determine risk factors for diabetic Charcot arthropathy were divided into three subgroups: sociodemographic profiles; foot factors; and diabetes characteristics.

Using the Neuropathy Symptom Score (NSS) questionnaire, face-to-face interviews were conducted during the patient's clinic visit to collect data on sociodemographic status, foot factors and severity of peripheral sensory neuropathy symptoms. ${ }^{(15)}$ Foot examinations were carried out to allocate patients to the case or control groups, as well as to determine the site of deformity and the site at which Semmes-Weinstein monofilament examination for peripheral neuropathy was to be performed. Data on the diabetes characteristics of all patients was retrieved from the patient medical records. Ethical approval from UMMC was obtained prior to the study. Verbal and written consent was also obtained from all patients before the commencement of the interview, and the collated information was kept confidential.

Data collected for the sociodemographic profile included age, gender, ethnicity, occupation and educational level. Age was calculated from the date of birth up to the year of the patient's first presentation with Charcot deformity for the case group, or taken during data collection for the control group. The date of birth was further verified by checking the patient's identification number. For the case group, the job in which the patient was engaged before the onset of Charcot foot deformity was taken as the occupation.

The patient's history of prior diabetic foot problems, such as foot ulcers, foot complex amputation, and/or other types of foot surgery (e.g. surgical debridement, osteotomy, fixation), was obtained either from the medical record or via patient self-report. The duration spent walking/standing per day and ambulation status were obtained from the patient self-report. For the case group, ambulation status referred to the ability to ambulate before the onset of Charcot deformity. Records on diabetes characteristics included type and duration of diabetes
Table I. Characteristics of diabetic Charcot foot presentation ( $n=48$ ).

\begin{tabular}{lr}
\hline Characteristic & No. (\%) \\
\hline Limb involvement & $40(83.3)$ \\
Unilateral & $8(16.7)$ \\
Bilateral & \\
Site of deformity & $22(45.8)$ \\
Midfoot & $11(22.9)$ \\
Ankle joint & $8(16.7)$ \\
Multiple sites & $5(10.4)$ \\
Hindfoot & $2(4.2)$ \\
Forefoot & \\
\hline
\end{tabular}

mellitus, medication, presence of retinopathy, nephropathy and peripheral neuropathy (on Semmes-Weinstein monofilament examination), symptom severity (evaluated using the NSS questionnaire), haemoglobin A1C (HbA1C) level, and body mass index (BMI). For the case group, these characteristics were determined prior to the diagnosis of diabetic Charcot arthropathy from available data in the patient medical record. A diagnosis of diabetic nephropathy was defined as deteriorating renal function with evidence of albuminuria. ${ }^{(16)}$ Retinopathy was defined as the presence of diabetic changes, as reported by an ophthalmologist in the patient medical record.

The sample size for the case and control groups, which met the minimum criteria, was estimated using statistical calculator software, OpenEpi version 2.3, for the unmatched case-control study on the basis of $95 \%$ confidence interval $(\mathrm{Cl})$ and $80 \%$ power. The case vs. control ratio was set at 1:1. All data was encoded and analysed using SPSS Statistics version 17.0 (SPSS Inc, Chicago, IL, USA). Chi-square test was used to test for statistical independence between the two groups. Inferential analysis with logistic regression was used to determine significant predictors of diabetic Charcot arthropathy. The final model for multiple logistic regression analysis included all variables with $\mathrm{p}<0.05$.

\section{RESULTS}

This retrospective case control study identified 48 diabetic patients with chronic diabetic foot Charcot arthropathy, comparing them with a control group of 52 diabetic patients who did not have Charcot arthropathy or other foot deformities. Table I summarises the characteristics of the patients with diabetic foot Charcot arthropathy. A large proportion of the diabetic patients presented with unilateral Charcot foot $(83.3 \%)$, with only $16.7 \%$ having bilateral foot involvement. The most common site of involvement of Charcot deformity was the midfoot, followed by the ankle joint, multiple sites, hindfoot and forefoot. All independent variables in the three subgroups (i.e. sociodemographic profiles, foot factors and diabetes characteristics) were compared between patients with and without diabetic Charcot arthropathy, and the results are summarised in Table II.

Up to $68.8 \%$ of patients with Charcot arthropathy were aged $<60$ (mean age $50.15 \pm 7.14$ ) years. Only a small percentage $(4.0 \%)$ of the cohort was aged $\leq 34$ years. In diabetic patients without Charcot arthropathy, the majority (67.3\%) were 
Table II. Sociodemographic details, foot factors and diabetes characteristics of patients.

\begin{tabular}{|c|c|c|c|c|c|}
\hline \multirow[t]{2}{*}{ Characteristic } & \multicolumn{2}{|c|}{$\%$} & \multirow[t]{2}{*}{ p-value } & \multirow{2}{*}{$\begin{array}{c}\text { Crude } \\
\text { OR }\end{array}$} & \multirow[t]{2}{*}{$95 \% \mathrm{Cl}$} \\
\hline & Case & Control & & & \\
\hline Age $<60 \mathrm{yr}^{*}$ & 68.8 & 32.7 & $<0.001$ & 4.5 & $1.9-10.5$ \\
\hline \multicolumn{6}{|l|}{ Gender } \\
\hline Male & 41.7 & 61.5 & 0.048 & 0.5 & $0.2-0.9$ \\
\hline Female & 58.3 & 38.5 & 0.048 & 2.2 & $1.0-4.9$ \\
\hline Working & 35.4 & 30.8 & 0.622 & 1.2 & $0.5-2.8$ \\
\hline Not working & 64.6 & 69.2 & 0.620 & 0.8 & $0.3-1.8$ \\
\hline \multicolumn{6}{|l|}{ Race } \\
\hline Malay & 43.8 & 28.8 & 0.261 & 1.6 & $0.7-3.8$ \\
\hline Indian & 50.0 & 53.8 & 0.260 & 0.6 & $0.2-1.4$ \\
\hline Chinese & 6.2 & 13.5 & 0.122 & 0.3 & $0.1-1.4$ \\
\hline \multicolumn{6}{|l|}{ Educational level } \\
\hline Tertiary & 27.0 & 29.0 & 0.490 & 0.6 & $0.2-2.0$ \\
\hline Secondary & 44.0 & 52.0 & 0.820 & 0.9 & $0.4-2.3$ \\
\hline Primary & 25.0 & 17.0 & 0.460 & 1.6 & $0.5-4.8$ \\
\hline History of diabetic foot problems ${ }^{+}$ & 77.1 & 15.4 & $<0.001$ & 18.5 & $6.7-50.8$ \\
\hline Prolonged walking $>5 \mathrm{hr}$ & 33.3 & 11.5 & 0.013 & 3.7 & $1.3-10.6$ \\
\hline Independent ambulator & 93.8 & 86.5 & 0.310 & - & - \\
\hline Duration of $\mathrm{DM}>10 \mathrm{yr}$ & 89.4 & 61.5 & 0.01 & 6.7 & $2.0-21.6$ \\
\hline Treatment with insulin/insulin + OHA & 87.2 & 50.0 & $<0.001$ & 6.8 & $2.5-18.8$ \\
\hline Retinopathy & 83.0 & 51.9 & 0.002 & 4.5 & $1.8-11.5$ \\
\hline Nephropathy & 62.8 & 23.5 & $<0.001$ & 5.4 & $2.2-13.4$ \\
\hline HbA1C > 6.5\% & 90.7 & 69.4 & 0.017 & 4.3 & $1.3-14.2$ \\
\hline No symptom & 10.6 & 21.2 & - & - & - \\
\hline Mild to severe & 89.4 & 78.8 & - & - & - \\
\hline
\end{tabular}

*Mean age $50.15 \pm 7.14$ yr. + Includes ulcer, surgery or ulcer and surgery. BMI: body mass index; Cl: confidence interval; DM: diabetes mellitus; HbA1C: haemoglobin A1C; NSS: Neuropathy Symptom Score; OHA: oral hypoglycaemic agents; OR: odds ratio

$>60$ years old. Univariate analysis showed that patients in the age group of $<60$ years were five times more likely to develop Charcot arthropathy (odds ratio [OR] 4.5; 95\% Cl 1.9-10.5). However, there were no significant associations between the development of Charcot arthropathy and gender, race, occupation or educational level.

The results also showed that $77.1 \%$ of patients with Charcot arthropathy had a history of prior foot problems that occurred in the foot with Charcot arthropathy, such as foot ulcer, amputation or surgery, or a combination of the aforementioned problems. Thus, patients with previous diabetic foot problems were 19 times more likely (OR 18.5; 95\% Cl 6.7-50.8) to develop Charcot arthropathy compared with the control group, where a large percentage $(84.6 \%)$ of patients did not have a history of foot problems. Table III shows the frequency and types of diabetic foot problems in the 48 patients prior to the onset of diabetic Charcot arthropathy. The most common type of foot problem in diabetics with Charcot arthropathy was foot ulcer, followed by a combination of ulcer and surgery. Foot surgery in this context referred to all types of surgery of the foot, including amputation of the foot complex, surgical debridement, incisional drainage and osteotomy for bony deformities.
Table III. Frequency and type of diabetic foot problems in patients with diabetic Charcot arthropathy $(n=48)$.

\begin{tabular}{lr}
\hline Parameter & No. (\%) \\
\hline Prior foot problems & $37(77.1)$ \\
Ulcer & $18(37.5)$ \\
Surgery & $8(16.7)$ \\
Ulcer + surgery & $11(22.9)$ \\
No prior foot problems & $11(22.9)$ \\
\hline
\end{tabular}

The majority of the patients with Charcot arthropathy (89.4\%) had had diabetes mellitus for more than ten years, as compared with the patients in the control group (61.5\%). Patients with chronic diabetes mellitus lasting more than ten years were seven times more likely to develop Charcot arthropathy (OR 6.7; 95\% Cl 2.0-21.6). The results also showed that insulin treatment, $\mathrm{HbA} 1 \mathrm{C}$ level $>6.5 \%$, and the presence of nephropathy and retinopathy were significant predictors of diabetic Charcot arthropathy (Table II). Up to $87.2 \%$ of patients with Charcot arthropathy were on either insulin or insulin with oral hypoglycaemic treatment, $83.0 \%$ had retinopathy, $62.8 \%$ had nephropathy and $90.7 \%$ had a $\mathrm{HbA} 1 \mathrm{C}$ level $>6.5 \%$. However, BMI $>23 \mathrm{~kg} / \mathrm{m}^{2} \mathrm{did}$ not appear to be a significant predictor $(p=0.198)$. 
Table IV. Adjusted odds ratio (OR) for diabetic Charcot arthropathy.

\begin{tabular}{lrlr}
\hline Characteristic & OR & 95\% Cl & p-value \\
\hline History of foot problems & 26.4 & $6.4-109.5$ & $<0.001$ \\
Duration of DM > 10 yr & 12.5 & $2.1-75.2$ & 0.006 \\
Age < 60 yr & 7.7 & $2.0-29.7$ & 0.003 \\
Nephropathy & 4.6 & $1.3-16.6$ & 0.018 \\
\hline
\end{tabular}

$\mathrm{Cl}$ : confidence interval; DM: diabetes mellitus

The presence of peripheral sensory neuropathy was established by the $10 \mathrm{~g}$ Semmes-Weinstein monofilament test, while symptoms of peripheral sensory neuropathy were elicited by the NSS questionnaire. ${ }^{(15)}$ Approximately $89.4 \%$ and $78.8 \%$ of the case and control groups, respectively, had symptoms of neuropathy (mild to severe). Most patients in the case and control groups (79.0\% vs. 58.0\%) experienced moderate to severe sensory neuropathy. In terms of the Semmes-Weinstein monofilament results, $80.0 \%$ of the control group had insensitivity (loss of protective sensation) compared to $96.4 \%$ in the case group.

To elucidate and determine the independent risk factors of diabetic Charcot arthropathy, multivariate analysis was performed using multiple logistic regression for all significant crude ORs. A history of prior foot problems (such as foot ulcer, amputation of foot complex, surgery or a combination of these) was found to be the most preponderant predictor of diabetic Charcot arthropathy, with a 26-time higher likelihood of development of Charcot arthropathy (OR 26.4; 95\% Cl 6.4-109.5). Other independent predictors identified in the present study were age $<60$ years, duration of diabetes mellitus $>10$ years and presence of nephropathy (Table IV).

\section{DISCUSSION}

The present study compared diabetic patients with chronic Charcot arthropathy of the foot to diabetic patients who did not have the condition. Comparisons of the patients sociodemographic profiles, diabetes characteristics and foot factors were made to predict the independent risk factors of diabetic Charcot arthropathy.

Our results showed that patients with a history of prior diabetic foot problems had the highest propensity for developing diabetic Charcot arthropathy. Other studies have similarly reported that a certain percentage of diabetic patients with Charcot arthropathy had a previous history of foot problems such as ulcer, surgery and/or amputation of the foot complex, along with a loss of protective sensation. ${ }^{(12,13,17)}$

Foot ulcer in diabetic patients with loss of protective sensation commonly occurs at the plantar aspect because of the abnormal high plantar pressure. Delayed management and the absence of adequate pressure offloading of the foot ulcer may further delay wound healing, instigate infection and perpetuate the progression of foot deformity. A non-healing, infected foot ulcer may require surgical debridement and amputation. ${ }^{(18)} \mathrm{A}$ few case reports have highlighted that a history of prior foot surgery, even without preceding foot ulcers, may instigate Charcot arthropathy as a result of altered weight-bearing forces, abnormal plantar pressure distribution and an ongoing inflammatory process. ${ }^{(19,20)}$
The common link that ongoing minor repetitive trauma and previous foot problems have with an insensate foot is that both act as triggering factors of inflammatory cascade and the continual production of proinflammatory cytokines, which can further accentuate the expression of receptor activator of the nuclear factor-kappa B ligand system. This phenomenon ultimately leads to osteolysis and osteopenia, resulting in bone breakdown..$^{(9,21,22)}$ Therefore, our finding of increased incidence of diabetic Charcot arthropathy in patients who have a history of foot problems is consistent with the novel inflammatory theory for the development of diabetic Charcot arthropathy. ${ }^{(9,21)}$

The most common site of Charcot arthropathy, based on the results of our study and literature search, is the midfoot, which involves the tarsal and/or tarso-metatarsal joints. ${ }^{(1,12,23)}$ Van also reported that midfoot deformity is the most common deformity seen in diabetic Charcot arthropathy with plantar ulceration complication and may predispose the patient to a long-term sequela of foot deformity. ${ }^{(24)}$

In the present study, analysis of diabetes characteristics suggests that chronicity of diabetes mellitus may show a predilection for Charcot arthropathy. Duration of diabetes mellitus of $>10$ years, insulin treatment, $\mathrm{HbA} 1 \mathrm{C}$ level $>6.5 \%$, and the presence of retinopathy and nephropathy are significantly associated with Charcot arthropathy $(p<0.05)$.

In a recent study on the risk factors of Charcot arthropathy, Nehring et al found that age appears to have a significant effect on patients with Charcot arthropathy as compared with Charcotfree patients. ${ }^{(25)}$ However, several studies have found that the younger age group is more prone to diabetic Charcot arthropathy than older people. ${ }^{(11-13)}$ This age trend is also consistent in our diabetic population, where the age group $<60$ (mean age $50.15 \pm 7.14$ ) years showed a significantly higher propensity for Charcot arthropathy.

There have been conflicting reports concerning the association of diabetic foot Charcot arthropathy with a younger age group and longer duration of diabetes mellitus (> 10 years). Our findings are similar to those of a large-scale study by Stuck et al, which reported that the incidence of diabetic foot Charcot arthropathy was higher among diabetics aged 55-64 years and those who have had diabetes mellitus for six years or more. ${ }^{(11)}$ The results of our study (i.e. the aforementioned two risk factors of diabetic foot Charcot arthropathy) should, however, be used with caution, due to the small sample size in this case-control study. It is possible that the diabetic population with Charcot arthropathy in our study was generally diagnosed at a younger age and, thus, developed micro- and macrovascular complications of diabetes mellitus earlier.

Being ambulant is one of the prerequisites for the development of Charcot arthropathy. ${ }^{(12)}$ Our study found that independence in ambulation alone is not a significant predictor of Charcot arthropathy; prolonged periods of walking or standing daily (at least more than five hours) also predisposed diabetic patients to Charcot arthropathy.

In the present study, both diabetics with and without Charcot arthropathy had a high incidence ( $80 \%)$ of peripheral 
sensory neuropathy symptoms, which can be mild to severe (based on NSS). The usual involvement of peripheral sensory neuropathy in diabetes mellitus is bilateral, but the most common presentation in Charcot arthropathy is unilateral at first onset. ${ }^{(12)}$ This natural history of diabetic Charcot arthropathy suggests that it is not a systemic pathology and that not all patients with diabetic peripheral neuropathy will eventually develop Charcot arthropathy.

Our study findings suggest that the interaction and combination of multiple factors (including history of prior diabetic foot problems, diabetes chronicity, age $<60$ years old, presence of nephropathy and retinopathy, and prolonged ambulation) further heightened the risk of development of diabetic Charcot arthropathy of the foot. Thus, it is important that preventive strategies in the management of diabetic foot problems involve a multidisciplinary team (e.g. podiatrist, primary care physician, endocrinologist, ophthalmologist and orthopaedic surgeon) to ensure early detection and optimum care. Risk stratification for diabetic foot may enable physicians and podiatrists to make better decisions on footcare management, intervals of follow-up, and the provision of offloading devices and protective footwear that prevent foot problems, thus reducing the incidence of new foot lesions and preserving the limb from amputation and deformity. ${ }^{(14)}$ Early detection and management with offloading devices and protective weight-bearing are also pertinent in preventing further bone destruction in Charcot arthropathy. ${ }^{(26)}$

The present study is not without limitations. First, the study recruited only patients with chronic Charcot arthropathy for the case group and retrospectively reviewed the predictors of Charcot arthropathy. Second, there may be possible bias due to missing data in the disease profile and recall bias when collecting information for the NSS questionnaire (such as history of diabetic foot problems and duration of ambulation), which may have affected the results. Furthermore, information from the time of onset of diabetic foot problems to the diagnosis of diabetic Charcot arthropathy was unavailable. To minimise the aforementioned bias, a larger-scale cohort study should be conducted in the future. Given that not all diabetic patients with peripheral neuropathy eventually develop diabetic Charcot arthropathy, further prospective studies with matched peripheral neuropathy groups should be conducted in order to focus on the predictors of this debilitating condition. Another limitation of the study was the absence of data on the history of foot problems in the contralateral foot, since the majority of the diabetic foot Charcot arthropathy patients presented with unilateral involvement. In future studies, this data should be included to strengthen the association of risk factors, since the presentation of diabetic foot Charcot arthropathy is commonly unilateral, while peripheral neuropathy usually presents with symmetrical involvement.

In conclusion, the present study has demonstrated that a history of diabetic foot problems, especially foot ulcers and a combination of foot ulcers and surgery of foot complex, independently elevates the risk of developing diabetic foot Charcot arthropathy. Other risk factors include a duration of diabetes mellitus longer than ten years, age less than 60 years and the presence of nephropathy. High vigilance toward the management of diabetic foot-care practices is of paramount importance for the prevention and early detection of diabetic foot problems. Therefore, using preventive strategies with diligent risk stratification, adequate offloading and plantar distribution management can reduce the incidence of diabetic foot problems. ${ }^{(14)}$ Diabetic Charcot arthropathy is associated with multifactorial risk factors and requires a concerted effort from multidisciplinary teams. Special scrutiny, foot care and education are imperative, especially in chronic diabetic patients with micro- and macrovascular complications. Further prospective research with matched peripheral neuropathy groups should be conducted.

\section{REFERENCES}

1. Jeffcoate W. The causes of the Charcot syndrome. Clin Podiatr Med Surg 2008; 25:29-42.

2. Frykberg RG, Belczyk R. Epidemiology of the Charcot foot. Clin Podiatr Med Surg 2008; 25:17-28.

3. Rajbhandari SM, Jenkins RC, Davies C, Tesfaye S. Charcot neuroarthropathy in diabetes mellitus. Diabetologia 2002; 45:1085-96

4. Gazis A, Pound N, Macfarlane R, et al. Mortality in patients with diabetic neuropathic osteoarthropathy (Charcot foot). Diabetic Med 2004; 21:1243-6.

5. Sohn MW, Lee TA, Stuck RM, Frykberg RG, Budiman-Mak E. Mortality risk of Charcot arthropathy compared with that of diabetic foot ulcer and diabetes alone. Diabetes Care 2009; 32:816-21.

6. Pakarinen TK, Laine HJ, Mäenpää H, Mattila P, Lahtela J. Long-term outcome and quality of life in patients with Charcot foot. Foot Ankle Surg 2009:187-91.

7. Jeffcoate W. Charcot neuro-osteoarthropathy. Diabetes Metab Res Rev 2008; 24 Suppl 1:S62-5.

8. Papanas N, Maltezos E. Etiology, pathophysiology and classifications of the diabetic Charcot foot. Diabet Foot Ankle 2013; 4.

9. Molines L, Darmon P, Raccah D. Charcot's foot: newest findings on its pathophysiology, diagnosis and treatment. Diabetes Metab 2010; 36:251-5.

10. Gouveri E, Papanas N. Charcot osteoarthropathy in diabetes: A brief review with an emphasis on clinical practice. World J Diabetes 2011; 2:59-65.

11. Stuck RM, Sohn MW, Budiman-Mak E, Lee TA, Weiss KB. Charcot arthropathy risk elevation in the obese diabetic population. Am J Med 2008; 121:1008-14.

12. Leung HB, Ho YC, Wong WC. Charcot Foot in a Hong Kong Chinese diabetic population. Hong Kong Med J 2009; 15:191-5.

13. Foltz KD, Fallat LM, Schwartz S. Usefulness of a brief assessment battery for early detection of Charcot foot deformity in patient with diabetes. J Foot Ankle Surg 2004; 43:87-92.

14. Frykberg RG, Zgonis T, Armstrong DG, et al. Diabetic Foot Disorders. A Clinical Practice Guideline (2006 revision). J Foot Ankle Surg 2006; 45(5 Suppl):S1-S66.

15. Abbott CA, Malik RA, van Ross ER, Kulkarni J, Boulton AJ. Prevalence and characteristics of painful diabetic neuropathy in a large community-based diabetic population in the U.K. Diabetes Care 2011; 34:2220-4.

16. Ministry of Health Malaysia. Clinical Practice Guideline: Diabetic Nephropathy. 2004. In: Academy of Medicine Malaysia [online]. Available at: http://www.acadmed.org.my/index.cfm?\&menuid=67. Accessed March 10, 2010.

17. Fabrin J, Larsen K, Holstein PE. Long-term follow-up in diabetic Charcot feet with spontaneous onset. Diabetes Care 2000; 23:796-800.

18. Ulbrecht JS, Cavanagh PR, Caputo GM. Foot problems in diabetes: an overview. Clin Infect Dis 2004; 39 Suppl 2:S73-82.

19. Fishco WD. Surgically induced Charcot's Foot. J Am Podiatr Med Assoc 2001; 91:388-93.

20. Aragón-Sánchez J, Lázaro-Martínez JL, Hernández-Herrero MJ. Triggering mechanisms of neuroarthropathy following conservative surgery for osteomyelitis. Diabetic Med 2010; 27:844-7.

21. Jeffcoate WJ, Game F, Cavanagh PR. The role of proinflammatory cytokines in the cause of neuropathic osteoarthropathy (acute Charcot foot) in diabetes. Lancet 2005; 366:2058-61. 
22. Mabilleau G, Petrova NL, Edmonds ME, Sabokbar A. Increased osteoclastic activity in acute Charcot's osteoarthopathy: the role of receptor activator of nuclear factor-kappaB ligand. Diabetologia 2008; 51:1035-40.

23. Herbst SA, Jones KB, Saltzman CL. Pattern of diabetic neuropathic arthropathy associated with the peripheral bone mineral density. J Bone Joint Surg Br 2004; 86:378-83.

24. Van GH. Rate of recurrence of plantar ulcer after wearing therapeutic footwear: 54 patients with diabetic Charcot Foot. Ann Phys Rehabil Med 2011; 54:e156.

25. Nehring P, Mrozikiewicz-Rakowska B, Maroszek P, et al. Risk factors of charcot neuroarthropathy development in patients with type 2 diabetes. Exp Clin Endocrinol Diabetes 2014; 122:31-4

26. Rogers LC, Frykberg RG, Armstrong DG, et al. The Charcot Foot in Diabetes. Diabetes Care 2011; 34:2123-9. 